\title{
ON THE FREIHEITSSATZ
}

\author{
ROGER C. LYNDON
}

\section{Summary}

The classical proof of the Dehn-Magnus Freiheitssatz, due to Magnus, is recast in the language of combinatorial geometry. This provides an extension of the Freiheitssatz from free groups to groups that are free products of groups isomorphic to subgroups of the additive group of real numbers, and, at the same time, a sharpening of the author's Identity Theorem.

\section{Introduction}

The following theorem is our main result.

THEOREM 1. Let $G$ be afree product of groups each isomorphic to a subgroup of the additive group of real numbers. Let $r$ be a cyclically reduced element of $G$, and let $w$ be a non-trivial element of the normal closure of $r$ in $G$. If the normal form for $r$ contains a factor from a certain one of the components, then so does the normal form for $w$.

This theorem clearly contains the classical Freiheitssatz, in which each component is an infinite cyclic group.

There is essentially only one proof known for the Freiheitssatz, that given by Magnus [5; see also 6]. Our starting point was an attempt to translate this proof into the language of combinatorial geometry, using a result of van Kampen [7] which we have discussed and exploited elsewhere [3]. (We have learned recently that Weinbaum [8] has already carried out a translation very similar to ours; however, his further applications of the method lie in a different direction from ours.) Our effort has been only partially successful. However, we were able to shift the emphasis of the proof, especially with regard to the induction argument, in such a way that it is easily adapted to prove the somewhat more general Theorem 1 above. The same argument without essential modification yields also a sharpened form of our Identity Theorem [1, 2], which we state as Theorem 4 below.

The result of van Kampen, as adapted to the group $G$, concerns what we have called diagrams. A diagram $M$ consists of a connected and simply connected finite complex $|M|$ in the plane together with a function $\phi$ assigning to each directed edge (1-cell) as label a non-trivial element of one of the component groups $G_{\alpha}$ of the free product $G$, and assigning inverse elements to oppositely oriented edges. The result of van Kampen asserts that if $R$ is any set of cyclically reduced elements of $G$, and $w$ any non-trivial element of the normal closure of $R$ in $G$, then there exists a diagram $M$ such that the product of the labels on the boundary of $M$, starting at a suitable point, is $w$, while, for any face (2-cell) of $M$, the product of the labels on the boundary of the face, starting at a suitable point, is $r$ or $r^{-1}$ for some element $r$ of $R$.

Received 11 July, 1970.

This work was done at Morehouse College, and was supported in part by the National Science Foundation. 
We may always suppose $M$ chosen with the smallest possible number of faces for given $R$ and $w$. Then $M$ will be reduced in the following sense. If $M_{1}$ is any subdiagram of $M$ containing at least one face but not all faces of $M$, then the product of the labels on $M_{1}$, starting at any point, does not reduce to the trivial element 1 of $G$.

In view of van Kampen's theorem, we can restate Theorem 1 in the following form.

THEOREM 2. Let $R$ consist of a single element $r$, let $w$ be a non-trivial element in the normal closure of $R$ in $G$, and let $M$ be a reduced diagram as above. If any edge of $M$ bears a label from a certain component $G_{\alpha}$, then some edge on the boundary of $M$ bears a label from that component.

Magnus' proof went by an induction, applied not to the Freiheitssatz itself, but to an extension of it. Similarly, we shall prove by induction an analogous extension of Theorem 2.

THEOREM 3. Let certain among the components $G_{\alpha}$ be denoted as $H_{a}, H_{a+1}, \ldots, H_{b}$, and suppose that $R$ consists of elements $r_{1}, r_{2}, \ldots, r_{m}$ where each $r_{i}$ contains at least one factor from some $H_{j}$. For each $r_{i}$ let $a_{i}$ be the least index $j$ such that $r_{i}$ contains a factor from $H_{j}$, and let $b_{i}$ be the greatest such index. Assume that $a_{1}<a_{2}<\ldots<a_{m}$ and $b_{1}<b_{2}<\ldots<b_{m}$. Let $w$ be a non-trivial element in the normal closure of $R$ in $G$, and let $M$ be a reduced diagram as above. If $j_{0}$ is the largest index such that some edge of $M$ bears a label from $H_{j_{0}}$, then some edge on the boundary of $M$ bears a label from $H_{j_{0}}$.

It is perhaps curious that, formulated thus, the extended Freiheitssatz takes the form of a combinatorial maximum principle (see [4]).

The main difference, although a minor one, between Magnus' argument and that to be given below seems to be that he argues by induction on the common length (in his case) of the $r_{i}$, while we argue by induction on the number of faces in the diagram $M$.

The planar diagrams $M$ considered above could just as well be taken as diagrams on the sphere, but not covering the entire sphere. If, now, we start with a diagram $M$ that exhausts the entire sphere, the arguments to be given below apply equally, and yield the following result.

THEOREM 4. Let $r$ be a cyclically reduced element of $G$. Then there does not exist a reduced diagram $M$ on the sphere such that $|M|$ is a decomposition of the entire sphere, and such that the product of the labels on each face, starting at a suitable point, is $r$ or $r^{-1}$.

Theorem 4, in the case in which $G$ is a free group, implies the Identity Theorem of the author $[1,2]$. Theorem 4 admits an obvious extension to the situation of Theorem 3.

\section{The inductive step for $m>1$}

We shall prove Theorem 3 by induction on the number of faces of $M$. The initial case, where $M$ has only a single face, is immediate. In this Section we prove Theorem 3 in the case in which $m>1$, assuming inductively that Theorem 3 holds for all diagrams 
with fewer faces than $M$. The remainder of the paper is devoted to the case in which $m=1$; the truth of Theorem 3 for given $M$ with $m=1$ will be reduced to that of Theorem 3 for a new diagram $M^{\prime \prime}$ with the same number of faces as $M$, but with $m>1$.

We assume then the hypotheses of Theorem 3, with $m>1$. To simplify notation, we may suppose that $M$ contains faces with labels $r_{1}^{ \pm 1}$ and $r_{m}^{ \pm 1}$. We must show that some edge on the boundary of $M$ bears a label from $\mathrm{H}_{b_{m}}$ (or, symmetrically, from $H_{a_{1}}$ ). In fact, we assume that no edge on the boundary bears a label from $H_{b_{m}}$, and from this derive a contradiction.

Let $M_{1}$ be a subcomplex of $M$, maximal with respect to the property that $M_{1}$ is connected and the union of a set of (closed) faces, each with boundary label $r_{m}{ }^{ \pm 1}$. Let $e$ be an edge on the boundary of $M_{1}$. If $e$ lies also on the boundary of $M$, then, by hypothesis, $e$ does not bear a label from $H_{b_{m}}$. Otherwise $e$ separates $M_{1}$ from a face $F$ that does not belong to $M_{1}$. From the maximality of $M_{1}$ we conclude that $F$ has boundary label $r_{i}^{ \pm 1}$ for some $i<m$. Since $r_{i}$ contains no factor from $H_{b_{m}}$, the label on $e$ does not come from $H_{b_{m}}$. We have shown that no edge on the boundary of $\mathrm{M}_{1}$ bears a label from $H_{b_{m}}$.

Suppose that $M_{1}$ is simply connected. Then $M_{1}$ is a subdiagram of $M$, containing at least one face but not all faces of $M$. Since $M$ is reduced, $M_{1}$ is reduced, and has non-trivial boundary label. This contradicts the induction hypothesis, according to which Theorem 3 holds for the smaller diagram $M_{1}$.

Suppose that $M_{1}$ is not simply connected. Then one of the components of the complement of $M_{1}$ in $M$ is a connected and simply connected subdiagram $M_{2}$ of $M$, with its boundary entirely contained in the boundary of $M_{1}$. From the maximality of $M_{1}$ it follows that $M_{2}$ contains a face with label $r_{i}^{ \pm 1}$ for some $i<m$; let $j$ be the least such $i$. Then $M_{2}$ contains an edge with label from $H_{a j}$. But every edge on the boundary of $M_{2}$ lies on the boundary of $M_{1}$, and hence on the boundary of some face $F$ of $M_{1}$; since the label $r_{m}^{ \pm 1}$ on $F$ contains no factor from $H_{a_{j}}$, the label on the edge cannot come from $H_{a j}$. This contradicts Theorem 3 (in the symmetric form noted above) for the smaller diagram $M_{2}$.

\section{The case $m=1$}

We assume that $M$ is given, with more than one face, and satisfying the hypotheses of Theorem 3 with $m=1$. We shall modify $M$ to obtain a new diagram $M^{\prime \prime}$ with the same number of faces, and satisfying the hypotheses of Theorem 3 with $m>1$. By the previous case, the conclusion of Theorem 3 follows for $M^{\prime \prime}$. We shall show that this implies the conclusion of Theorem 3 for $M$.

It is easy to see that the case $m=1$ of Theorem 3 is equivalent to Theorem 2 . We assume that $G, r, w$, and $M$ are as in the hypothesis of Theorem 2 .

Let $\mu$ be any homomorphism from $G$ into $\mathbb{R}$, the additive group of reals, such that $\mu(r)=0$. If $\pi$ is any path in $M$, let $S(\pi)$ be the sum of values of $\mu$ on the labels on the edges of $\pi$. The condition $\mu(r)=0$ implies that $S(\pi)$ depends only on the endpoints of $\pi$. Choose a vertex $v_{0}$ in $M$ for base point, and, for each vertex $v$ in $M$, define $h(v)$ to be the common value of $S(\pi)$ for all paths $\pi$ from $v_{0}$ to $v$. Without loss of generality we can suppose that each component $G_{\alpha}$ of $G$ is isomorphic to $\mathbb{R}$, and that a standard 
isomorphism $\gamma_{\alpha}$ from $\mathbb{R}$ on to $G_{\alpha}$ is given. With this we can extend the function $h$ by linearity to interior points of the edges of $M$.

Let $K$ be the finite set of values assumed by the function $h$ at the vertices of $M$; we can suppose the elements of $K$ are $k_{1}<k_{2}<\ldots<k_{n}$. If, on a given edge $e$, the function $h$ is not constant, it is strictly monotonic, hence assumes each of the values $k_{i}$ at most once. We modify our diagram $M$ by introducing as new vertices all those points on $e$ where $h$ assumes a value $k_{i}$ from $K$. Each edge $e$ is now subdivided into a succession of edges $e_{1}, e_{2}, \ldots, e_{t}$. Using the functions $\gamma_{\alpha}$ and linearity, we can factor the label $g$ on $e$ into labels $g_{1}, g_{2}, \ldots, g_{t}$ for $e_{1}, e_{2}, \ldots, e_{t}$. We have thus obtained a new diagram $M^{\prime}$, with the same number of faces as $M$.

The group $G$ is the free product of components $G_{\alpha}$, for $\alpha$ in some index set $A$. We define a new group $G^{\prime \prime}$ to be the free product of components $G_{\alpha, k}$, for $\alpha$ in $A$ and $k$ in $K$; we suppose given isomorphisms $\gamma_{\alpha, k}$ from $G_{\alpha}$ onto $G_{\alpha, k}$. If $h$ is constant on an edge $e$, then $h(e)=k_{i}$ for some $k_{i}$ in $K$; otherwise $h(e)=\left[k_{i}, k_{i+1}\right]$ for some $k_{i}$. In either case we define $k(e)=k_{i}$. Now let $e$ be an edge, directed in such a way that its label $g$ is 'positive', that is, if $g$ is in $G_{\alpha}$, then $\gamma_{\alpha}{ }^{-1}(g)$ is a positive element in $\mathbb{R}$. We replace the label $g$ on $e$ by the new label $\gamma_{\alpha, k(e)}(g)$ in $G_{\alpha, k(e)}$. We have thus a new diagram $\mathrm{M}^{\prime \prime}$, with labels from the group $G^{\prime \prime}$; it is evident that $M^{\prime \prime}$ is reduced and has non-trivial boundary label. We shall next examine the labels on the faces of $M^{\prime \prime}$.

Let $F_{1}$ and $F_{2}$ be two faces of $M$, with $p_{1}$ a vertex on the boundary of $F_{1}$ and $p_{2}$ on $F_{2}$. We shall say $p_{1}$ and $p_{2}$ are corresponding points if the boundary labels, on $F_{1}$ beginning at $p_{1}$ and on $F_{2}$ beginning at $p_{2}$, are the same or inverses. (Apart from the case in which $r$ is a proper power, which can be excluded, $p_{1}$ uniquely determines $p_{2}$.) Let $q_{1}$ and $q_{2}$ be another pair of corresponding points. Then the path along the boundary of $F_{1}$ from $p_{1}$ to $q_{1}$, in the positive or negative sense according as the label on $F_{1}$ is $r$ or $r^{-1}$, and the similar path on the boundary of $F_{2}$, will bear the same product-label, say $u$. From this we have that $h\left(q_{1}\right)=h\left(p_{1}\right)+\mu(u)$ and $h\left(q_{2}\right)=h\left(p_{2}\right)+\mu(u)$.

It follows that $h\left(q_{2}\right)-h\left(q_{1}\right)=h\left(p_{2}\right)-h\left(p_{1}\right)$. Thus there exists a real number $d$, depending only on $F_{1}$ and $F_{2}$, such that if $p_{1}$ and $p_{2}$ are any two corresponding points, $p_{1}$ on $F_{1}$ and $p_{2}$ on $F_{2}$, then $h\left(p_{2}\right)=h\left(p_{1}\right)+d$. Similarly, if $e_{1}$ and $e_{2}$ are corresponding edges, that is beginning at corresponding points, and have positive labels, then $k\left(e_{2}\right)=k\left(e_{1}\right)+d$. Now, in the new diagram $M^{\prime \prime}, F_{1}$ and $F_{2}$ will have boundary labels $r_{1}{ }^{ \pm 1}$ and $r_{2}{ }^{ \pm 1}$, where $r_{1}$ and $r_{2}$ are obtained from $r$ by replacing each factor of $r$, from some $G_{\alpha,}$, by a corresponding factor from some $G_{\alpha, k}$. From the above we see that $r_{2}$ is obtainable from $r_{1}$ by replacing each factor of $r_{1}$, from some $G_{\alpha, k}$, by the corresponding factor from $G_{\alpha, k+d}$. We now arrange the finite set of $r_{i}$, arising as above, in order: $r_{1}, r_{2}, \ldots, r_{m}$, in such a way that in passing from an earlier $r_{i}$ to a later $r_{j}$, the second subscript on the component $G_{\alpha, k}$ containing corresponding factors is always increased.

We now show that if Theorem 2 failed for $M$, then Theorem 3 would fail for $M^{\prime \prime}$. Suppose Theorem 2 fails for $M$. Then $M$ contains an edge with a label from a certain $G_{\beta}$, but no such edge on its boundary. Now $M^{\prime \prime}$ will contain edges with labels from some of the $G_{\beta, k}$, but no such edge on its boundary. Let $H_{1}=G_{\beta, k_{1}}$, $H_{2}=G_{\beta, k_{1}}, \ldots, H_{n}=G_{\beta, k_{n}}$. The hypotheses of Theorem 3 are evidently satisfied, but the conclusion fails flagrantly. Now, provided we know that $m>1$, this is a contradiction, and the proof is completed. 
The next Section is devoted to showing that $\mu$ can always be chosen in'such a way as to yield $m>1$.

\section{Completion of the proof}

Let $G, r, w$, and $M$ be, as before, as in the hypothesis of Theorem 2 . We want to show that it is always possible to choose $\mu$ in such a way that the construction above yields $M^{\prime \prime}$ with $m>1$.

There is in fact one degenerate case where $\mu$ cannot be so chosen; we dispose of this case by a direct argument. Let $r$ be called special if it is of the form $r=\left(g_{1} g_{2} \ldots g_{t}\right)^{n}$ for some $t \geqslant 1$ and $n \geqslant 1$, and with each $g_{i}$ from a different $G_{\alpha_{i}}$. We assume that no boundary edge of $M$ has a label from, say, $G_{\beta}=G_{\alpha_{1}}$, and will derive a contradiction. Note that this assumption implies that $t \geqslant 2$. Let $B$ be that part of the 1-skeleton of $M$ consisting of all edges with labels from $G_{\beta}$; then $B$ is non-empty and contains no edge from the boundary of $M$. If any component of $B$ were a tree, it would contain a vertex $v$ with a single edge $e$ of $B$ emanating from it. Since $e$ is not on the boundary of $M$, it separates two faces $F_{1}$ and $F_{2}$. Now it is easy to see that the subdiagram $M_{1}=F_{1} \cup F_{2}$ violates the assumption that $M$ is reduced. We conclude that $B$ contains a circuit, and hence that some component of the complement of $B$ in $M$ is a subdiagram $M_{2}$ with boundary contained entirely in $B$. It follows that no edge on the boundary of $M_{2}$ bears a label from $G_{\alpha_{2}}$. Since $M_{2}$ is smaller than $M$, we can conclude by induction that this is impossible.

A point on $r$ splits $r$ into two parts: $r=u v$, product without cancellation; formally, we define a point $a$ to be an ordered couple $a=(u, v)$ such that $r=u v$ without cancellation. Let $p$ be a vertex of $M$, lying on the boundary of a face $F$. Then the boundary label of $F$, beginning at $p$, is $s^{ \pm 1}$ for some cyclic conjugate $s$ of $r$; now, for some $u$ and $v$, we have $r=u v$ and $s=v u$. We say that $p$ determines the point $a=(u, v)$ on $r$ relative to $F$. It is clear how to extend this definition to the case in which $p$ is an interior point of an edge. Let $p$ lie on an edge separating faces $F_{1}$ and $F_{2}$. Then $p$ determines two points $a_{1}$ and $a_{2}$ on $r$, relative to $F_{1}$ and $F_{2}$. We say that $a_{1}$ and $a_{2}$ are immediately related. We call two points $a$ and $b$ on $r$ related if they are connected by a chain with consecutive members immediately related. We call two factors of $r$ related (immediately related) if they contain interior points that are related (immediately related).

Suppose that, for some component $G_{\alpha}$, not all the factors of $r$ from $G_{\alpha}$ are related. Then we can divide these factors into two sets $S_{1}$ and $S_{2}$ such that no factor in $S_{1}$ is related to a factor in $S_{2}$. We modify $G$ to $G^{\prime}$ by replacing the component $G_{\alpha}$ by two components $G_{\alpha, 1}$ and $G_{\alpha, 2}$. If an edge $e$ of $M$ separates faces $F_{1}$ and $F_{2}$, and has label from $G_{\alpha}$, then the two factors of $r$ that it determines relative to $F_{1}$ and $F_{2}$ are related and so belong to the same set $S_{i}$; we replace the label on $e$ by the corresponding element of $G_{\alpha, i}$. We have now a new diagram $M^{\prime}$, all of whose faces have label $r^{ \pm 1}$, where $r^{\prime}$ is obtained from $r$ by replacing each factor from $S_{i}$ by the corresponding element of $G_{\alpha, i}$. If Theorem 2 holds for $M^{\prime}$, then it holds also for $M$. Finite iteration of this argument permits us to confine attention to the case in which all factors of $r$ from the same component are related.

We return to a point $p$ on an edge separating two faces $F_{1}$ and $F_{2}$, and the points $a_{1}$ and $a_{2}$ of $r$ determined by $p$ relative to faces $F_{1}$ and $F_{2}$. Let $v$ be the segment of $r$ (or a cyclic conjugate) running from $a_{1}$ to $a_{2}$; that is, suppose $r=u v z$ with $a_{1}=(u, v z)$ and $a_{2}=(u v, z)$. Suppose $\mu$ has been chosen so that $\mu(v) \neq 0$. The 
point $a_{1}$ on $r$ will be determined by a point $q$ on the boundary of $F_{2}$, and the segment of the boundary of $F_{2}$ running from $q$ to $p$ will bear label $v$, whence

$$
h(p)=h(q)+\mu(v) \neq h(q) .
$$

Thus the corresponding points $p$ on $F_{1}$ and $q$ on $F_{2}$ receive different values of $h$, and it follows that, in $M^{\prime \prime}, F_{1}$ and $F_{2}$ will bear labels $r_{i}{ }^{ \pm 1}$ for different $i$, whence $m>1$. Suppose now that we have a chain $a_{0}, a_{1}, \ldots, a_{n}$ of points on $r$, with each pair $a_{i}, a_{i+1}$ immediately related. We may write $a_{i}=\left(u v_{1} v_{2} \ldots v_{i}, v_{i+1} \ldots v_{n} z\right)$, hence $a_{0}=(u, v z)$ and $a_{n}=(u v, z)$ for $v=v_{1} v_{2} \ldots v_{n}$. Now if $\mu(v) \neq 0$, then some $\mu\left(v_{i}\right) \neq 0$, and $m>1$ as before. To obtain $m>1$ then it suffices to choose $\mu$ so that $\mu(v) \neq 0$ where $v$ is the segment of $r$ determined by two related points of $r$.

Write $G=G_{1}{ }^{*} G_{2}{ }^{*} \ldots{ }^{*} G_{n}$. Using the given isomorphisms $\gamma_{\alpha}$ from $\mathbb{R}$ onto the $G_{\alpha}$, we can write each element of $G_{\alpha}$ as $\gamma_{\alpha}(x)$, for $x$ in $\mathbb{R}$. Clearly a homomorphism $\mu$ from $G$ into $\mathbb{R}$ is uniquely determined by its values $\mu_{\alpha}=\mu\left(\gamma_{\alpha}(1)\right)$. We can identify the commutator quotient group $\bar{G}$ of $G$ with the direct product of the components, $\bar{G}=G_{1} \times G_{2} \times \ldots \times G_{n}$. Let the image of $r$ in $G$ be $r=\gamma_{1}\left(x_{1}\right) \gamma_{2}\left(x_{2}\right) \ldots \gamma_{n}\left(x_{n}\right)$. Then $\mu(r)=0$ if and only if $\Sigma \mu_{\alpha} x_{\alpha}=0$.

We call a component $G_{\alpha}$ idle if $x_{\alpha}=0$, and otherwise engaged. Evidently, to choose $\mu$ with $\mu(r)=0$, we may choose $\mu_{\alpha}$ arbitrarily for all idle $G_{\alpha}$, and also for all but one of the engaged $G_{\alpha}$. Suppose first that $r$ contains a factor from some idle component $G_{\alpha}$. Then $r$ must contain at least two factors $g$ and $h$ from $G_{\alpha}$, and, choosing successive such factors, $r$ has a part $g u h$ where $u$ is a product of factors none of which lies in $G_{\alpha}$. We may now suppose this configuration chosen with as few factors in $u$ as possible; then $u$ does not contain two factors from the same idle component. Suppose $u$ contained a single factor $f$ from an idle component, say $u=u_{1} f u_{2}$. Then, by choosing $\mu(f)$ arbitrarily we make $\mu(u)$ arbitrary. Now if $a$ in $g$ and $b$ in $h$ are related points in $g$ and $h$, we have in $r$ a part $v$ from $a$ to $b$, where $v=g_{2} u h_{1}, g=g_{1} g_{2}$, $h=h_{1} h_{2}$. If $\mu(u)$ is arbitrary, then so is $\mu(v)$; in particular, we can make $\mu(v) \neq 0$, and the proof is complete.

Suppose next that $u$ contains no factor from an idle component. Again $\mu(u)$ is arbitrary, and the proof is complete, unless $u$ contains factors from all the engaged components. Let $k$ be the next factor in $r$ from $G_{\alpha}$ after $h$; here we take $r$ cyclically, and it may happen that $k$ is $g$. Then $r$ has a part $h z k$. If $z$ contains a factor $f_{2}$ from an engaged component, then $u$ also contains a factor $f_{1}$ from this component, and $r$ has a part $f_{1} q f_{2}$, where $q$ contains the single factor $h$ from $G_{\alpha}$. Since we can choose $\mu(h)$, and so $\mu(q)$, arbitrarily, the proof is complete as before. The case remains in which all factors from $z$ are idle. If we now choose a shortest segment $h z k$ consisting of idle factors only, and with $h$ and $k$ factors from the same component, then $z$ must contain a single factor $f$ from some idle component. Now $\mu(f)$, and so $\mu(z)$, is arbitrary, and again the proof is complete. This completes the case in which $r$ contains any factor from an idle component.

We may suppose now that all components are engaged. Suppose $r$ contains two factors $g$ and $h$ from the same component, $G_{\alpha}$ and hence a part $g u h$. Unless $u$ contains a factor from each component other than $G_{\alpha}$, we may choose $\mu\left(g_{2} u h_{1}\right)$ arbitrarily, and the proof is complete. Thus we may assume that in $r$, between two factors from the same component there occurs a factor from each other component. This implies 
that $r$ has the form

$$
r=\left(\gamma_{1}\left(x_{11}\right) \gamma_{2}\left(x_{12}\right) \ldots \gamma_{n}\left(x_{1 n}\right)\right)\left(\gamma_{1}\left(x_{21}\right) \ldots\right) \ldots\left(\gamma_{1}\left(x_{t 1}\right) \gamma_{2}\left(x_{t 2}\right) \ldots \gamma_{n}\left(x_{t n}\right)\right)
$$

for some $t \geqslant 1$. We may put aside the case $n=1$, where the theorem to be proved is trivial, and also the case $t=1$, where $r$ is special. Now, taking $\gamma_{1}\left(x_{11}\right)$ and $\gamma_{1}\left(x_{21}\right)$ in the role of $g$ and $h$, we can still make $\mu\left(g_{2} u h_{1}\right) \neq 0$ unless $x_{12}, x_{13}, \ldots x_{1 n}$ are proportional to $x_{2}, x_{3}, \ldots, x_{n}$, say $x_{12}=c_{1} x_{2}, x_{13}=c_{1} x_{3}, \ldots, x_{1 n}=c_{1} x_{n}$. Next, taking $\gamma_{2}\left(x_{12}\right)$ and $\gamma_{2}\left(x_{22}\right)$ as $g$ and $h$, we have, for some $c_{2}$, that

$$
x_{13}=c_{2} x_{3}, x_{14}=c_{2} x_{4}, \ldots, x_{21}=c_{2} x_{1} \text {. }
$$

If $n \geqslant 3$, we have $x_{13}=c_{1} x_{3}=c_{2} x_{3}$, whence $c_{1}=c_{2}=c$. Continuation of this argument shows that each $x_{i j}=c x_{j}$, whence

and $r$ is special.

$$
r=\left(\gamma_{1}\left(c x_{1}\right) \gamma_{2}\left(c x_{2}\right) \ldots \gamma_{n}\left(c x_{n}\right)\right)^{t}
$$

The case remains in which $n=2$, and this is a genuine exception to the argument above. To treat this case we remark first that, if there are two related points in the same factor, then the segment separating them lies in one of the components; then $\mu$ can be chosen not to vanish on this segment, and the proof is complete. We assume now that there are not two related points in the same factor. Pick a point $a$ of $r$, interior to some factor, and a small segment $u$ of that factor containing $a$ in its interior. In each other factor from the same component $G_{\alpha}$, the set of points related to points in $u$ will be a finite union of intervals (possibly empty). We introduce all end points of such intervals, on all faces of $M$, as new vertices, and we relabel such intervals with elements from a new component $G_{\alpha, 1}$. We have thus replaced $M$ by a new diagram $\mathrm{M}^{\prime}$ with labels $r^{\prime \pm 1}$ where $r^{\prime}$, in a new group $G^{\prime}$, has factors from three components. Evidently Theorem 2 for $M^{\prime}$ implies the same for $M$. Thus we have reduced the case $n=2$ to the case $n \geqslant 3$, already treated. This completes the proof of the theorem.

\section{References}

1. R. C. Lyndon, "Cohomology theory of groups with a single defining relation ", Ann. of Math., 52 (1950), 650-665.

2. - "Dependence and independence in free groups", J. reine angew. Math., 210 (1962), 148-174.

3. _ـ, "On Dehn's algorithm", Math. Ann., 166 (1966), 208-228.

4. _- "A maximum principle for graphs", J. Comb. Theory, 3 (1967), 34-37.

5. W. Magnus, "Uber diskontinuerliche Gruppen mit einer definierenden Relation (Der Freiheitssatz)", J. reine angew. Math., 103 (1930), 141-165.

6. - A. Karrass, and D. Solitar, Combinatorial group theory (Interscience, Wiley, New York, 1966).

7. E. R. van Kampen, "On some lemmas in the theory of groups", Amer. J. Math., 55 (1933), 268-273.

8. C. M. Weinbaum, "On relators and diagrams for groups with one defining relation" (to appear).

University of Michigan,

Ann Arbor,

Michigan 48104. 